\title{
Imaging Hippocampal Function across the Human Life Span: Is Memory Decline Normal or Not?
}

\author{
Scott A. Small, MD, ${ }^{1,3}$ Wei Yann Tsai, PhD, ${ }^{2}$ Robert DeLaPaz, MD, ${ }^{4}$ Richard Mayeux, MD, ${ }^{1,3}$ \\ and Yaakov Stern, $\mathrm{PhD}^{1,3}$
}

Memory function commonly declines in later life. Whether memory decline represents a disease process or whether it is part of normal aging remains unknown. Here we answer this question by assessing the function of multiple subregions that make up the hippocampal circuit across the human life span. A newly developed MRI approach-designed to detect functional changes in individual hippocampal subregions-was used to assess the hippocampal circuit in 70 subjects between 20 and 88 years of age. Using strict parametric criteria, analysis revealed that function in two hippocampal subregions - the subiculum and the dentate gyrus - decline normally with age. In contrast, function in the entorhinal cortex declines pathologically. Single-subject analysis revealed that hippocampal dysfunction, found in $60 \%$ of elders was selectively correlated with memory decline. These results show that memory decline is caused by different mechanisms and suggests how memory decline should be approached clinically.

Ann Neurol 2002;51:290-295

DOI 10.1002/ana.10105

Higher cortical function is a dynamic process that changes across the human life span. ${ }^{1,2}$ Late in life, memory function is particularly sensitive to the effects of aging, and memory loss is one of the most common complaints among the elderly. Whether age-related memory decline is normal remains unknown because older individuals are also susceptible to diseases that target memory regions of the brain. Should the diminished ability to acquire new information be viewed as part of normal senescence, as the tail end of the aging trajectory mirroring early development, or does memory decline reflect a disease process? This is a fundamental question for establishing the full scope of normal cortical aging and for characterizing age-related disease, and the answer will dictate whether age-related memory decline be considered a clinical entity. If so, as larger segments of the population are entering late life, memory decline among the elderly will emerge as a significant public health concern.

The hippocampal formation is important for forming new long-term episodic memories ${ }^{3}$ and is targeted by diseases such as Alzheimer's disease as well as by numerous physiologic processes that change in an agedependent manner. ${ }^{4}$ Other forms of memory function, such as working memory, are subserved by alternative brain regions, such as the prefrontal cortex. ${ }^{5}$ The hippocampal formation is not a simple structure but rather is made up of separate anatomic subregionsthe entorhinal cortex, the dentate gyrus, the CA subfields, and the subiculum. ${ }^{6}$ Because the neuronal populations within each subregion have unique molecular phenotypes, the subregions are differentially vulnerable to mechanisms of dysfunction. For example, the entorhinal cortex is the first subregion targeted by Alzheimer's pathology, ${ }^{7}$ the CA1 subregion is sensitive to cerebrovascular changes, ${ }^{8}$ and the dentate gyrus is sensitive to changes in hormonal levels.

An important aspect of hippocampal physiology is that the subregions do not function independently. Because the subregions are interconnected the hippocampus functions as a circuit, so that lesions to any subregion interrupts global hippocampal function and produces overlapping deficits in memory performance. ${ }^{10}$ Dissociating the various causes of age-related memory decline, therefore, cannot be achieved by methods that assess global hippocampal function, such as neuropsychological testing. Furthermore, measurements of global hippocampal function cannot determine which
From the ${ }^{1}$ The Taub Institute on Alzheimer's Disease and the Aging Brain, ${ }^{2}$ School of Public Health, and the Departments of ${ }^{3} \mathrm{Neu}-$ rology and ${ }^{4}$ Radiology, Columbia University College of Physicians and Surgeons, New York, NY.

Received Jun 26, 2001, and in revised form Nov 1. Accepted for publication Nov 5, 2001.
Address correspondence to Dr Small, Columbia University, College of Physicians and Surgeons, PH 19th Floor, 630 West 168 Street, New York, NY 10032. E-mail: sas68@columbia.edu 
subregion undergoes normal age-related changes from others that are targeted by disease processes. Resolving this issue requires a method that assesses the function of the hippocampal subregions individually and simultaneously, thereby capturing circuit properties of the hippocampus. The absence of such of method, to a large extent, accounts for the continued controversy about the nature and causes of age-related memory decline.

With these goals in mind, we have recently developed an application of magnetic resonance imaging (MRI) designed to assess the functional integrity of individual hippocampal subregions. ${ }^{11}$ This approach relies on the paramagnetic properties of deoxyhemoglo$\operatorname{bin}^{12,13}$ and the putative relationship between basal metabolism and hemodynamic variables - such as oxygen extraction fraction-which are correlates of basal deoxyhemoglobin concentrations. Thus, dysfunctional subregions are expected to have lower basal metabolism, higher deoxyhemoglobin concentration, and lower T2*-weighted signal compared to the same subregion with normal function. Indeed, we have previously found that this resting oxygen (ROXY) approach can detect hippocampal dysfunction in patients with $\mathrm{Alz}$ heimer's dementia ${ }^{15}$ and in transgenic mice who phenocopy memory decline. ${ }^{11}$ Here we used this imaging approach to evaluate the hippocampal circuit in a large group of healthy individuals across the life span, sampled from two populations.

There are no "gold standard" serological or histological markers of disease that can be used as a clear indication of hippocampal pathology. Even early Alzheimer's pathology, an undisputed cause of memory decline, results in changes in hippocampal physiology prior to cell loss or to the histological markers of disease. ${ }^{16}$ Making the distinction between normal and pathological changes in the hippocampal formation has to rely, therefore, on evaluating neuronal function directly, ${ }^{17}$ mapping the patterns of change in neuronal function over time. ${ }^{18}$

The effect of normal aging on the brain is, by definition, a stochastic process that targets all individuals in a population ${ }^{19}$; in contrast, the effects of age-related disease are nonrandom and target a subpopulation of individuals. Accordingly, two parametric criteria should be met if age-related changes in brain function are normal: Brain function should decline in a continuous fashion across the life span and, more importantly, the variance of brain function among an older age group should be equal to the variance among a younger age group, although a shift in the mean is expected.

\section{Patients and Methods}

Patients were recruited from two populations. Forty younger individuals ranging from 20 to 62 years of age were recruited from the population of Columbia University employees. All individuals had normal medical and neurological exams. Thirty older individuals ranging from 70 to 88 years of age were recruited from an aging project in which a random sample of community-based individuals over the age of 65 is followed longitudinally, with subjects receiving annual medical and neuropsychological evaluations. ${ }^{20}$ Older patients recruited for this study were followed for at least 5 years, during which time they were healthy-they were not diagnosed with dementia, stroke, Parkinson's disease, or depressionand their performance on neuropsychological testing was within normal limits. The older individuals participated in a previously reported study. ${ }^{11}$ All patients gave informed consent.

\section{Imaging}

A sagittal T1-weighted image was acquired to identify the long axis of the hippocampal formation. Five $5 \mathrm{~mm}$ oblique coronal slices were selected perpendicular to long axis of the hippocampal formation. The most anterior slice was always placed at the posterior aspect of the amygdala. Foam pads and surgical tape were used to secure the head. Patients were instructed to close their eyes, and scanning was performed using a 1.5 tesla scanner. GRASS gradient-echo T2*weighted images were acquired $(\mathrm{TE}=45 \mathrm{~ms}$, TR $=300 \mathrm{~ms}$; flip angle $=10$ degrees; number of excitations $=3$; in-plane resolution $=0.86 \mathrm{~mm} \times 0.86 \mathrm{~mm}$ ).

Postacquisition data processing was performed on a Silicon Graphics Indigo II work station, using image display and analysis software packages (IDL Research Systems). Data analysis was performed on a PC-based linux workstation using MEDx (Sensor Systems, Inc.) and was performed by an investigator blind to subject grouping.

A standard slice was selected just anterior to the lateral geniculate nucleus that provided optimal visualization of anatomical landmarks - the general morphology of the hippocampus and the internal white matter tracts-and that was anterior enough to include the entorhinal cortex. ${ }^{21}$ Standard atlases were used ${ }^{21,22}$ to identify landmarks localizing the general locale of the hippocampal subregions, and within each subregion four contiguous pixels with the greatest signal was selected and averaged, and then divided by the global signal measured from the slice. A logarithmic transformation was applied to the ratios, assuring that the measurements were normally distributed. Because of the inability to determine the precise circumference of the hippocampal subregions, without the aid of histological staining, ${ }^{21}$ volumetrics of the subregions could not be determined.

\section{Data Analysis}

Two approaches were used to determine whether age-related changes in hippocampal signal are normal. In the first approach a linear regression model was used to test for a relationship between signal and age in any subregion. Group was included as a covariate because the patients were selected from separate populations. In the second approach Levene's test was used to test for equality of variance in signal among the oldest patients, those in their $70 \mathrm{~s}$ and $80 \mathrm{~s}$, and the youngest patients, those in their 20 s and 30 s.

The final series of analysis were performed to establish the frequency of age-related hippocampal decline in the elderly 
and to validate that the MRI signal reflects neuronal function. The hippocampal circuit of each older subject was compared to the youngest age group using a multivariate linear regression, where the independent variables were the four hippocampal subregions and the dependent variable was group. Patients were dichotomized into those in whom hippocampal signal did not significantly differ from the younger age group and those who had at least one hippocampal subregion showing significant decline in signal, thereby establishing the observed frequency of elders with age-related hippocampal decline. Because patients received repeated neuropsychological testing, rates of change in cognitive function were determined for each subject. Memory was assessed with the Selective Reminding Test, ${ }^{23}$ abstract reasoning was assessed with Wechsler Adult Intelligence Scale-Revised similarities, ${ }^{24}$ and language ability was assessed with the Controlled word association task. ${ }^{25}$ Slopes were calculated by subtracting last performance from first performance and dividing by time. In order to validate that hippocampal signal reflected hippocampal function, a multivariate analysis of variance was performed comparing the patients with and without normal hippocampal signal, where the slopes from the three cognitive domains were included as the dependent variables.

\section{Results}

The demographics of the young and old cohorts are shown in the Table. A larger proportion of patients in the older group were women compared to the younger group ( $81 \%$ vs $\left.51 \% ; \chi^{2}=7.2, p \leq 0.01\right)$; however, gender did not influence MRI signal intensity.

Results revealed that signal declined in all hippocampal subregions but, importantly, the pattern of decline differed across the subregions (Figs 1 and 2). For both the dentate gyrus and the subiculum, a linear regression analysis showed that signal declined continuously across the life span, independent of age group (dentate gyrus, beta $=-0.65, p \leq 0.01$; subiculum, beta $=$ $0.5, p \leq 0.05)$. A continuous decline in signal was observed even when the younger age-group was evaluated alone (dentate gyrus, beta $=-0.4, p \leq 0.01$; subiculum, beta $=-0.32, p \leq 0.05)$. In contrast, signal in the entorhinal cortex declined in a discontinuous fashion, characterized by a step function in the older group (beta $=-0.6, p \leq 0.05)$, independent of age (beta $=$

Table. Demographics of the Young and Old Cohort of Subjects

\begin{tabular}{lcc}
\hline & Young & Old \\
\hline Age (mean and range) & $40(21-62$ yrs $)$ & $79(70-88 \mathrm{yrs})$ \\
$\begin{array}{l}\text { Gender (\% women) } \\
\text { Education (mean yrs) }\end{array}$ & $50 \%$ & $80 \%$ \\
$\begin{array}{l}\text { Ethnicity (percent } \\
\text { nonwhite) }\end{array}$ & $70 \%$ & 12 \\
$\begin{array}{l}\text { APOE 4 (\% with at } \\
\text { least one allele) }\end{array}$ & na & $35 \%$ \\
\hline
\end{tabular}

na $=$ not applicable.
0.16, $p \leq 0.5)$. Entorhinal signal was not correlated with age when the younger age group was evaluated alone (beta $=0.12$ ). Beyond the global effect of the regression model neither age (beta $=-0.24$ ) nor group (beta $=-0.12$ ) were correlated with signal measured from the CA1 subregion.

The difference in patterns of decline appeared to reflect an age-related increase in variability in entorhinal signal, with only a subgroup of elders having lower signal intensity, whereas the variance of signal from the dentate gyrus and subiculum appeared stable (see Figs 1 and 2). This was tested formally by comparing the variance of signal among the oldest patients - those in their 70s and 80s-to the variance of signal in the youngest patients- those in their 20s and 30s. Indeed, signal variance from the dentate gyrus $(\mathrm{F}=1.8, p=$ 0.2 ) and the subiculum ( $F=0.48, p=0.5)$ were equal between both groups, whereas signal variance from the entorhinal cortex was significantly greater among older individuals $(\mathrm{F}=4.3, p \leq 0.05)$.

Although memory decline is common in later life, it is not inevitable. In order to determine the frequency of hippocampal decline among the older patients, a multivariate linear regression model was used comparing hippocampal signal for each older subject compared to the group of younger patients. Eighteen $(60 \%)$ elders had significantly diminished signal in at least one hippocampal subregion; 12 (40\%) showed no decline in hippocampal signal. Among the elders with hippocampal decline, 7 (23\%) individuals had predominant decline in the subiculum or dentate gyrus and 7 $(23 \%)$ had predominant decline in the entorhinal cortex (Fig 3).

Older patients with and without hippocampal dysfunction were compared on cognitive performance to test whether signal reflects memory decline. Results revealed that patients with hippocampal dysfunction had greater decline in memory over time $(\mathrm{F}=5.4, p \leq$ $0.05)$ but were not different on measures of language $(\mathrm{F}=0.84)$ or abstract reasoning $(\mathrm{F}=0.76)$ (Fig 4). A cognitive comparison between the 7 patients with diminished signal in the subiculum or dentate gyrus to the 7 patients with diminished entorhinal signal revealed no significant difference $(\mathrm{F}=1.1)$.

\section{Discussion}

This is first study to explore how the function of the hippocampal subregions varies across the human life span. Postmortem series have assessed the structure of the hippocampus and have documented age-related cell loss in select hippocampal subregions. ${ }^{26,27}$ Many agerelated processes that target the hippocampal formation, however, cause hippocampal dysfunction by interrupting the normal physiology of hippocampal neurons, without necessarily resulting in structural damage. ${ }^{16,28}$ The need to evaluate the function not 

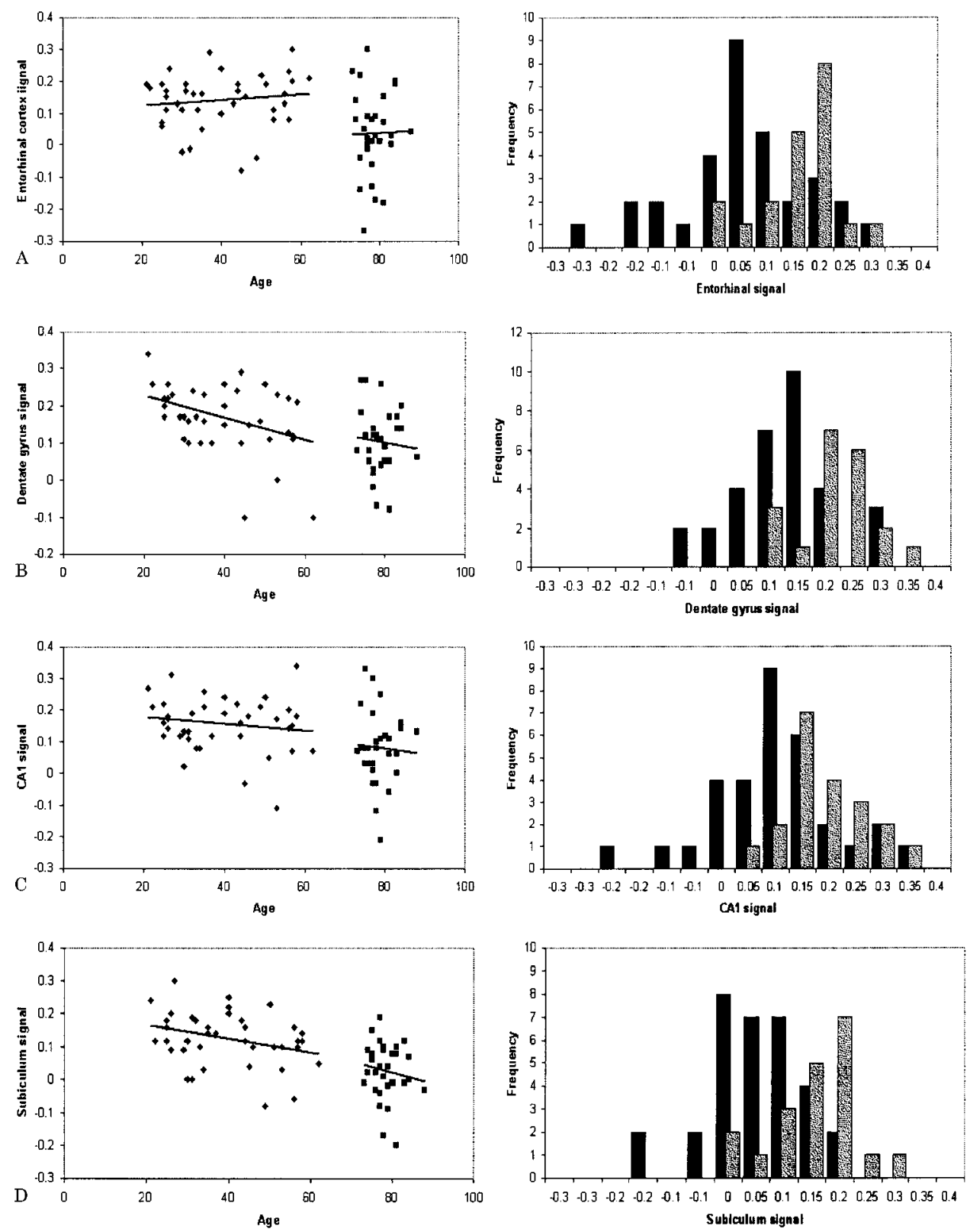

Fig 1. Hippocampal signal declines over the life span, but different patterns of decline were found in separate hippocampal subregions. (A) The entorhinal cortex; (B) the dentate gyrus; (C) CA1; and (D) the subiculum. Scatter plots show the relationship between signal and age, and linear regression lines were fitted separately for the younger and older groups. Histograms show the distribution of signal in the oldest age group (70-90 years) (dark bars) and youngest age group (20-40 years) (light bars). The entorbinal cortex fulfilled criteria for pathological decline, whereas the subiculum and the dentate gyrus fulfilled criteria for normal decline.

structure of neurons has been highlighted in animal studies where age-related memory decline is associated with physiologic lesions to hippocampal neuronalie, in the absence of cell loss. ${ }^{29}$ In a series of studies with genetically modified mice, the imaging technique used here was sensitive enough to detect changes in neuronal physiology, even in the absence of cell loss or other structural lesions. ${ }^{11}$ Using this method we provide direct evidence that hippocampal function de- clines with age, and that diminished hippocampal function-reflecting cell number or cell physiologyunderlies age-related memory decline.

The more important utility of this method is that it allowed us to rigorously address whether memory decline is normal by assessing patterns of decline in individual hippocampal subregions, the basic processing unit of the hippocampal circuit. The main finding of this study showed that a decline in entorhinal signal is 

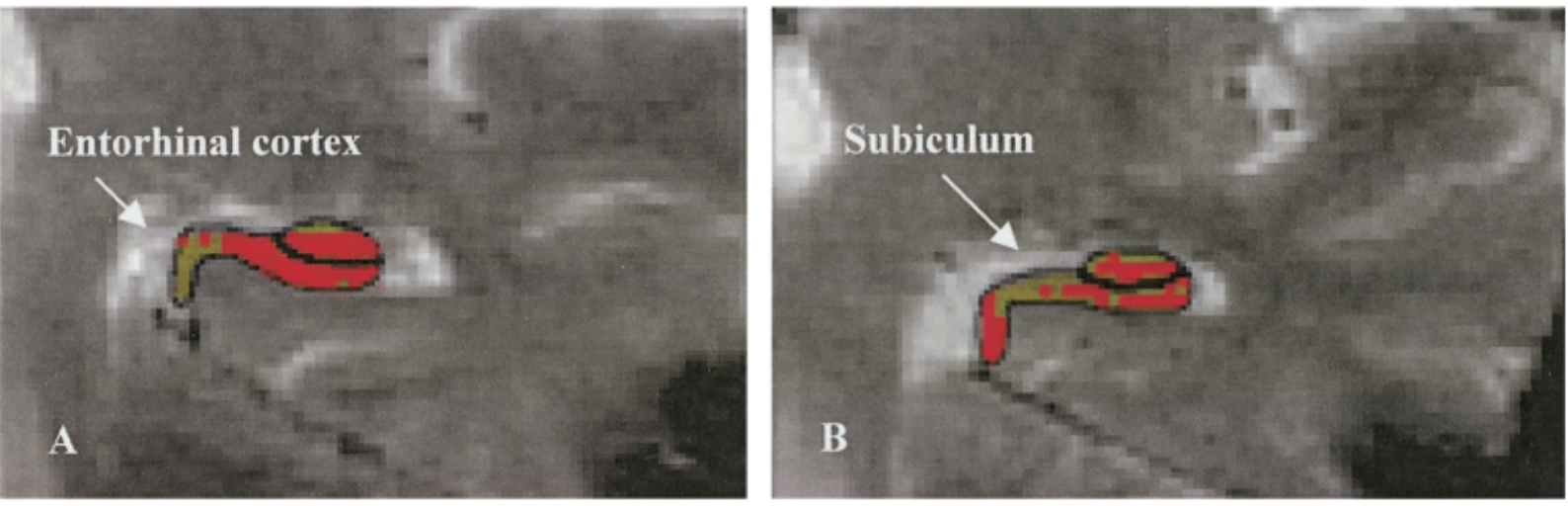

Fig 2. Hippocampal dysfunction localizes to different subregions; individual examples. High-resolution T2*-weighted magnetic resonance images were acquired through the hippocampal formation, and a standard slice was selected anterior to the lateral geniculate nucleus. The external morphology of the hippocampus and the internal white matter tracts (black lines) were used as landmarks to identify the general locale of the subregions. Normalized signal was color-coded in a dichotomous fashion to illustrate locations of dysfunction, where red $\geq 1$ and green $<1$. Hippocampal dysfunction was localized to the entorhinal cortex in a 78-year-old woman and to the subiculum in an 81-year-old man. EC = entorhinal cortex; SUB = subiculum; DG $=$ dentate gyrus.

not normal, and therefore memory decline in individuals with entorhinal dysfunction likely reflects a disease process. In contrast, the pattern of signal decline in the subiculum and the dentate gyrus is consistent with normal aging. A mixed picture was found in the CA1 subregion, suggesting that the CA1 subregion is targeted by both normal and pathological causes of agerelated decline. All forms of hippocampal decline produced similar cognitive deficits-with selective decline in memory performance-highlighting circuit properties of the hippocampal formation.

Twenty-three percent of the community-based 70and 80-year-olds had the pathological form of hippocampal dysfunction. Although the precise prevalence needs to be established in a larger cohort, the observed frequency is likely an underestimate. Subjects who were healthy and nondemented at baseline were excluded from consideration if they were found to have demen-

Fig 3. Hippocampal dysfunction localized to different subregions; group data. The hippocampal circuit of each healthy elder was compared to the hippocampal circuit of a group of younger individuals. Of the 30 elderly patients, 18 were found to have diminished hippocampal signal and 12 had stable hippocampal signal. The 18 elders with age-related diminished signal were then subdivided based on the subregion with the greatest dysfunction.

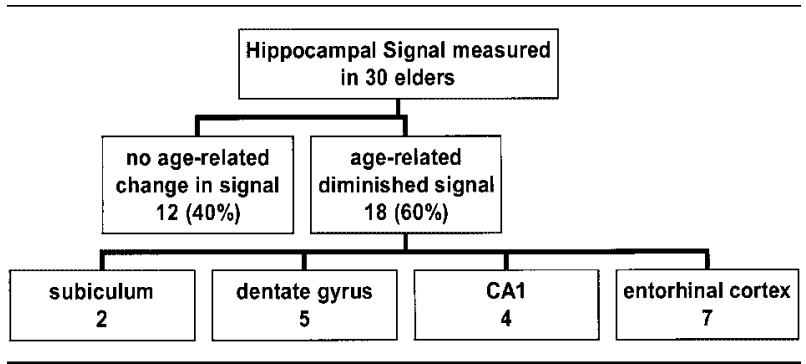

tia at follow-up. This allowed us to use longitudinal measures of cognition, providing a more precise metric of cognitive decline compared to single time-point evaluations but biased against elderly individuals with incipient dementia. Furthermore, some of the individuals with changes in the CA1 subregion likely have the pathological form of decline. Even if the frequency of elders with pathological memory decline is a conservative estimate, the results suggest that it occurs in almost a quarter of the aging population studied. Thus, at the very least, this form of age-related memory decline represents a serious public health concern.

The full complement of causes underlying agerelated hippocampal dysfunction has not been established but the list includes Alzheimer's pathology, agerelated changes in hormonal levels and cerebrovascular supply, and age-related accrual of oxidative stress. ${ }^{4} \mathrm{Ge}-$ netic factors also play a role in cognitive function

Fig 4. Hippocampal dysfunction is selectively associated with memory decline. Compared to elders with preserved hippocampal function (dark bars), elders with hippocampal dysfunction (light bars) had a greater decline in memory performance but not in language ability or abstract reasoning.

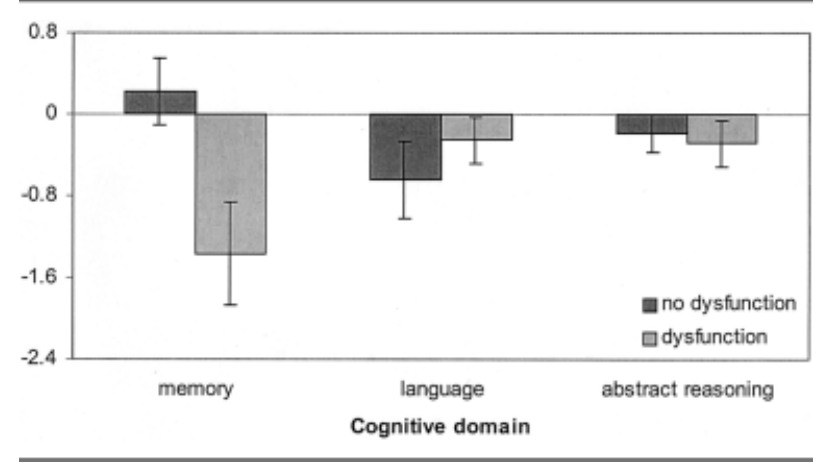


among the elderly, and possibly influence repair mechanisms in the setting of injury to hippocampal neurons. The entorhinal cortex is the first hippocampal subregion targeted by Alzheimer's pathology, and the pathological decline observed in the signal of the entorhinal cortex likely reflects elders with the earliest manifestations of Alzheimer's pathology. If so, individuals with entorhinal dysfunction are at increased risk to progress to Alzheimer's dementia, and we are currently testing this prediction by following subjects prospectively.

In summary, this study provides primary evidence that the function of the human hippocampus declines over the life span, and an analysis of the hippocampus compatible with its anatomical complexity reveals separate mechanisms of decline: normal and pathological. These findings should inform investigators interested in understanding the dynamic range and the mechanisms that mediate normal cortical aging by focusing their attention on appropriate neuronal populations within the hippocampal circuit. Furthermore, these findings should inform clinicians interested in diagnosing and developing treatments for age-related brain disease. Most important, perhaps, these findings put an end to the ongoing debate whether age-related memory decline is a legitimate clinical entity. Shifting societal perspectives on aging have influenced how late-life cognitive changes have been viewed clinically. On the one extreme, rigorous analysis of Alzheimer's disease was delayed late into the last century because "senility" was implicitly accepted as normal aging during a more parochial era. On the other extreme, our era is too quick to categorize any change associated with aging as abnormal on practical grounds that even a subtle change interferes with demanding daily lives. In this study we bypass these debates by providing objective biological evidence that for many individuals in their 7 th and 8th decades memory decline reflects a pathological state.

This work was supported in part by federal grants (AG08702 and AG00949 to SAS) and the Beeson Faculty Scholar Award from the American Federation of Aging (SAS).

\section{References}

1. Petersen RC, Smith G, Kokmen E, et al. Memory function in normal aging. Neurology 1992;42:396-401.

2. Small SA, Stern Y, Tang M, Mayeux R. Selective decline in memory function among healthy elderly. Neurology 1999;52: 1392-1396.

3. Squire LR. Memory and the hippocampus: a synthesis from findings with rats, monkeys, and humans [published erratum appears in Psychol Rev 1992;99:582]. Psychol Rev 1992;99: 195-231.

4. Small SA. Age-related memory decline: current concepts and future directions. Arch Neurol 2001;58:360-364.

5. Postle BR, Berger JS, Taich AM, D'Esposito M. Activity in human frontal cortex associated with spatial working memory and saccadic behavior. J Cogn Neurosci 2000;12(Suppl 2):2-14.
6. Amaral DG, Witter MP. The three-dimensional organization of the hippocampal formation: a review of anatomical data. Neuroscience 1989;31:571-591.

7. Braak H, Braak E. Evolution of the neuropathology of Alzheimer's disease. Acta Neurol Scand Suppl 1996;165:3-12.

8. Dela Torre JC, Fortin T, Park GA, et al. Chronic cerebrovascular insufficiency induces dementia-like deficits in aged rats. Brain Res 1992;582:186-195.

9. Galea LA, McEwen BS. Sex and seasonal differences in the rate of cell proliferation in the dentate gyrus of adult wild meadow voles. Neuroscience 1999;89:955-964.

10. Jarrad LE. On the role of the hippocampus in learning and memory in the rat. Behav Neural Biol 1993;60:9-26.

11. Small S, Wu E. Bartsch D, et al. Imaging physiologic dysfunction of individual hippocampal subregions in humans and genetically modified mice. Neuron 2000;28:653-664.

12. Thulborn KR, Waterson JC, Matthews PM, Radda GK. Oxygen dependence of the transverse relaxation time of water protons in whole blood at high field. Biochimica et Biophysica Acta 1982;714:265-270.

13. Ogawa S, Lee TM, Nayak AS, Glynn P. Oxygenation-sensitive contrast in magnetic resonance imaging of rodent brain at high magnetic fields. Magn Reson Med 1990;14:68-78.

14. Raichle ME, MacLeod AM, Snyder AZ, et al. A default mode of brain function. Proc Natl Acad Sci U S A 2001;98: 676-682.

15. Small SA, Nava AS, Perera GM, et al. Evaluating the function of hippocampal subregions with high-resolution MRI in Alzheimer's disease and aging. Microsc Res Tech 2000;51:101-108.

16. Hsia AY, Masliah E, McConlogue L, et al. Plaque-dependent disruption of neural circuits in Alzheimer's disease mouse models. Proc Natl Acad Sci U S A 1999;96:3228-3233.

17. Barnes CA. Normal aging: regionally specific changes in hippocampal synaptic transmission. Trends Neurosci 1994;17: $13-18$.

18. Rapp PR, Amaral DG. Individual differences in the cognitive and neurobiological consequences of normal aging. Trends Neurosci 1992;15:340-345.

19. Hayflick L. The future of aging. Nature 2000;408:267-269.

20. Small SA, Perera GM, DeLaPaz R, et al. Differential regional dysfunction of the hippocampal formation among elderly with memory decline and Alzheimer's disease. Ann Neurol 1999;45: 466-472.

21. Amaral DG, Insausti R. The hippocampal formation. In: Paxinos R, ed. The human nervous system. San Diego: Academic, 1990.

22. Duvernoy HM. The human hippocampus: an atlas of applied anatomy. 2nd ed. Munich: Bergman, 1998.

23. Buschke H, Fuld PA. Evaluating storage, retention, and retrieval in disordered memory and learning. Neurology 1974;24: 1019-1025.

24. Wechsler D. WAIS-R manual. New York: The Psychological Corp., 1981

25. Benton AL. FAS test. Neurosensory Center comprehensive examination for aphasia. Victoria, British Columbia: University of Victoria, 1967.

26. West MJ. Regionally specific loss of neurons in the aging human hippocampus. Neurobiol Aging 1993;14:287-293.

27. Simic G, Kostovic I, Winblad, et al. Volume and number of neurons of the hippocampal formation in normal aging and Alzheimer's disease. J Comp Neurol 1997;379:482-494.

28. McEwen BS. Cortiscosteroids and hippocampal plasticity. Ann N Y Acad Sci 1994;746:134-142; discussion 142-144, 178179.

29. Rapp PR, Gallagher M. Preserved neuron number in the hippocampus of aged rats with spatial learning deficits. Proc Natl Acad Sci U S A 1996;93:9926-9930. 